Table S1. Emission standards for on-road vehicles in the EU and Russia.

\begin{tabular}{llllll}
\hline $\begin{array}{l}\text { Passenger } \\
\text { cars* }\end{array}$ & $\begin{array}{l}\text { Introduced } \\
\text { in the EU }\end{array}$ & $\begin{array}{l}\text { Introduced } \\
\text { in Russia }\end{array}$ & $\begin{array}{l}\text { Heavy-duty diesel } \\
\text { engines }\end{array}$ & $\begin{array}{l}\text { Introduced } \\
\text { in the EU }\end{array}$ & $\begin{array}{l}\text { Introduced } \\
\text { in Russia }\end{array}$ \\
\hline Euro 1 & 1992 & - & Euro I & 1992 & - \\
Euro 2 & 1996 & 2006 & Euro II & 1996 & 2006 \\
Euro 3 & 2000 & 2008 & Euro III & 2000 & 2008 \\
Euro 4 & 2005 & 2013 & Euro IV & 2005 & 2013 \\
Euro 5 & 2009 & 2014 & Euro V & 2008 & 2016 \\
Euro 6 & 2014 & n/a & Euro VI & 2013 & n/a \\
\hline
\end{tabular}

* By convention, light-duty vehicles are marked with Arabic numerals while Roman numbers are used for heavyduty vehicles (trucks and buses).

Table S2. COPERT and NIIAT emission factors and BC speciation for hot operation stage.

\begin{tabular}{lcccccccccc}
\hline Type & Subcategory & \multicolumn{7}{c}{ COPERT EF, g/km } & \multicolumn{7}{c}{ Blended } & NIIAT EFs, g/km & \multirow{2}{*}{ EC/PM* } & \multirow{2}{*}{ OC/EC* } \\
\hline Cars & & Urban & \multicolumn{2}{c}{ Rural } & Highway & Urban & Rural & Highway & & \\
\hline Euro 0 & & 0.271 & 0.199 & 0.146 & 0.250 & 0.150 & 0.170 & 0.55 & 0.70 \\
Euro 1 & & 0.07 & 0.057 & 0.087 & 0.073 & 0.040 & 0.050 & 0.70 & 0.40 \\
Euro 2 & & 0.058 & 0.047 & 0.045 & 0.073 & 0.040 & 0.050 & 0.80 & 0.23 \\
Euro 3 & & 0.035 & 0.029 & 0.038 & 0.053 & 0.030 & 0.030 & 0.85 & 0.15 \\
Euro 4 & & 0.034 & 0.029 & 0.025 & 0.016 & 0.090 & 0.090 & 0.87 & 0.13 \\
Euro 5 & & 0.003 & 0.002 & 0.002 & 0.004 & 0.002 & 0.002 & 0.20 & 2.00 \\
\hline LCV & & & & & & & & & \\
Euro 0 & & 0.281 & 0.285 & 0.337 & 0.290 & 0.210 & 0.230 & 0.55 & 0.70 \\
Euro 1 & & 0.099 & 0.07 & 0.118 & 0.087 & 0.060 & 0.100 & 0.70 & 0.40 \\
Euro 2 & & 0.099 & 0.07 & 0.118 & 0.087 & 0.060 & 0.100 & 0.80 & 0.23 \\
Euro 3 & & 0.066 & 0.047 & 0.079 & 0.057 & 0.040 & 0.060 & 0.85 & 0.15 \\
Euro 4 & & 0.035 & 0.024 & 0.041 & 0.033 & 0.020 & 0.030 & 0.87 & 0.13 \\
Euro 5 & & 0.002 & 0.001 & 0.001 & 0.002 & 0.001 & 0.002 & 0.20 & 2.00 \\
Trucks & & & & & & & & & \\
Euro 0 & $<=7,5 \mathrm{t}$ & 0.4 & 0.297 & 0.211 & 0.543 & 0.180 & 0.180 & 0.50 & 0.80 \\
Euro I & $<=7,5 \mathrm{t}$ & 0.157 & 0.116 & 0.09 & 0.360 & 0.140 & 0.140 & 0.65 & 0.40 \\
Euro II & $<=7,5 \mathrm{t}$ & 0.069 & 0.056 & 0.064 & 0.220 & 0.080 & 0.080 & 0.65 & 0.40 \\
Euro III & $<=7,5 \mathrm{t}$ & 0.082 & 0.061 & 0.04 & 0.153 & 0.060 & 0.060 & 0.70 & 0.30 \\
Euro IV & $<=7,5 \mathrm{t}$ & 0.017 & 0.014 & 0.015 & 0.030 & 0.010 & 0.010 & 0.75 & 0.25 \\
Euro V & $<=7,5 \mathrm{t}$ & 0.021 & 0.018 & 0.015 & 0.030 & 0.010 & 0.010 & 0.75 & 0.25 \\
Euro 0 & $7,5-12 \mathrm{t}$ & 0.423 & 0.301 & 0.201 & 0.893 & 0.400 & 0.400 & 0.50 & 0.80 \\
Euro I & $7,5-12 \mathrm{t}$ & 0.262 & 0.182 & 0.129 & 0.640 & 0.330 & 0.330 & 0.65 & 0.40 \\
Euro II & $7,5-12 \mathrm{t}$ & 0.115 & 0.087 & 0.098 & 0.230 & 0.100 & 0.100 & 0.65 & 0.40 \\
Euro III & $7,5-12 \mathrm{t}$ & 0.133 & 0.095 & 0.062 & 0.153 & 0.060 & 0.060 & 0.70 & 0.30 \\
Euro IV & $7,5-12 \mathrm{t}$ & 0.027 & 0.021 & 0.02 & 0.030 & 0.010 & 0.010 & 0.75 & 0.25 \\
Euro V & $7,5-12 \mathrm{t}$ & 0.034 & 0.026 & 0.021 & 0.030 & 0.010 & 0.010 & 0.75 & 0.25 \\
& & & & & 1 & & & & &
\end{tabular}




\begin{tabular}{|c|c|c|c|c|c|c|c|c|c|}
\hline Euro 0 & $12-14 t$ & 0.452 & 0.32 & 0.232 & 1.073 & 0.550 & 0.550 & 0.50 & 0.80 \\
\hline Euro I & $12-14 t$ & 0.28 & 0.199 & 0.147 & 0.697 & 0.480 & 0.480 & 0.65 & 0.40 \\
\hline Euro II & $12-14 t$ & 0.128 & 0.095 & 0.109 & 0.310 & 0.180 & 0.180 & 0.65 & 0.40 \\
\hline Euro III & $12-14 t$ & 0.141 & 0.099 & 0.071 & 0.193 & 0.130 & 0.130 & 0.70 & 0.30 \\
\hline Euro IV & $12-14 t$ & 0.03 & 0.023 & 0.02 & 0.040 & 0.020 & 0.020 & 0.75 & 0.25 \\
\hline Euro V & $12-14 t$ & 0.036 & 0.028 & 0.023 & 0.040 & 0.020 & 0.020 & 0.75 & 0.25 \\
\hline Euro 0 & $>14 \mathrm{t}$ & 0.625 & 0.439 & 0.29 & 1.073 & 0.550 & 0.550 & 0.50 & 0.80 \\
\hline Euro I & $>14 \mathrm{t}$ & 0.386 & 0.271 & 0.175 & 0.697 & 0.480 & 0.480 & 0.65 & 0.40 \\
\hline Euro II & $>14 \mathrm{t}$ & 0.164 & 0.118 & 0.129 & 0.310 & 0.180 & 0.180 & 0.65 & 0.40 \\
\hline Euro III & $>14 \mathrm{t}$ & 0.199 & 0.139 & 0.087 & 0.193 & 0.130 & 0.130 & 0.70 & 0.30 \\
\hline Euro IV & $>14 \mathrm{t}$ & 0.039 & 0.029 & 0.023 & 0.040 & 0.020 & 0.020 & 0.75 & 0.25 \\
\hline Euro V & $>14 \mathrm{t}$ & 0.049 & 0.037 & 0.028 & 0.040 & 0.020 & 0.020 & 0.75 & 0.25 \\
\hline \multicolumn{10}{|l|}{ Buses } \\
\hline Euro 0 & $<=15 \mathrm{t}$ & 0.858 & 0.574 & 0.388 & 0.880 & 0.270 & 0.295 & 0.50 & 0.80 \\
\hline Euro I & $<=15 \mathrm{t}$ & 0.294 & 0.221 & 0.173 & 0.650 & 0.215 & 0.230 & 0.65 & 0.40 \\
\hline Euro II & $<=15 \mathrm{t}$ & 0.142 & 0.114 & 0.107 & 0.398 & 0.195 & 0.175 & 0.65 & 0.40 \\
\hline Euro III & $<=15 \mathrm{t}$ & 0.146 & 0.11 & 0.099 & 0.197 & 0.105 & 0.100 & 0.70 & 0.30 \\
\hline Euro IV & $<=15 \mathrm{t}$ & 0.035 & 0.027 & 0.022 & 0.040 & 0.025 & 0.025 & 0.75 & 0.25 \\
\hline Euro V & $<=15 \mathrm{t}$ & 0.042 & 0.031 & 0.038 & 0.037 & 0.025 & 0.025 & 0.75 & 0.25 \\
\hline Euro 0 & $15-18 t$ & 0.767 & 0.52 & 0.312 & 1.523 & 0.430 & 0.500 & 0.50 & 0.80 \\
\hline Euro I & $15-18 t$ & 0.412 & 0.294 & 0.217 & 0.890 & 0.310 & 0.400 & 0.65 & 0.40 \\
\hline Euro II & $15-18 t$ & 0.197 & 0.157 & 0.138 & 0.680 & 0.310 & 0.270 & 0.65 & 0.40 \\
\hline Euro III & $15-18 \mathrm{t}$ & 0.195 & 0.148 & 0.108 & 0.250 & 0.130 & 0.120 & 0.70 & 0.30 \\
\hline Euro IV & $15-18 \mathrm{t}$ & 0.049 & 0.037 & 0.027 & 0.050 & 0.030 & 0.030 & 0.75 & 0.25 \\
\hline Euro V & $15-18 t$ & 0.055 & 0.042 & 0.036 & 0.050 & 0.030 & 0.030 & 0.75 & 0.25 \\
\hline Euro 0 & $>18 \mathrm{t}$ & 0.957 & 0.675 & 0.395 & 1.523 & 0.430 & 0.500 & 0.50 & 0.80 \\
\hline Euro I & $>18 \mathrm{t}$ & 0.517 & 0.37 & 0.227 & 0.757 & 0.310 & 0.400 & 0.65 & 0.40 \\
\hline Euro II & $>18 \mathrm{t}$ & 0.265 & 0.212 & 0.174 & 0.583 & 0.310 & 0.270 & 0.65 & 0.40 \\
\hline Euro III & $>18 \mathrm{t}$ & 0.24 & 0.17 & 0.125 & 0.250 & 0.130 & 0.120 & 0.70 & 0.30 \\
\hline Euro IV & $>18 \mathrm{t}$ & 0.06 & 0.045 & 0.029 & 0.050 & 0.030 & 0.030 & 0.75 & 0.25 \\
\hline Euro V & $>18 \mathrm{t}$ & 0.066 & 0.049 & 0.04 & 0.050 & 0.030 & 0.030 & 0.75 & 0.25 \\
\hline
\end{tabular}

Sources: (Emisia, 2015; NIIAT, 2012).

* EC/PM and OC/EC speciation factors are derived from the COPERT model. 
Table S3. Number of active diesel vehicles by type and emission standard in Russia, 2014.

\begin{tabular}{lrrrr}
\hline & Cars & LCVs & Trucks & Buses \\
\hline Euro 0 & 100620 & 150345 & 470737 & 24994 \\
Euro 1 & 103529 & 119732 & 43093 & 10038 \\
Euro 2 & 113576 & 94135 & 173337 & 17541 \\
Euro 3 & 144298 & 140923 & 293520 & 49725 \\
Euro 4 & 691199 & 353189 & 271189 & 19481 \\
Euro 5 & $329941^{*}$ & 58703 & 77404 & 1615 \\
Total & 1483163 & 917027 & 1329280 & 123394 \\
\hline
\end{tabular}

* - includes 2110 Euro 6 cars

Calculated based on (Avtostat, 2015).

Table S4. The annual average distance traveled by type of vehicles, thousand $\mathrm{kmyr}^{-1}$.

\begin{tabular}{|l|l|l|l|l|}
\hline & Cars & LCVs & Trucks & Buses \\
\hline NIIAT (1998) & $\begin{array}{l}\text { 15 } \\
10 \text { for 5-year old } \\
\text { Russian cars } \\
10 \text { for 10-year old } \\
\text { foreign cars }\end{array}$ & & $\begin{array}{l}35 \text { in cities } \\
60 \text { suburban } \\
100 \text { intercity }\end{array}$ & $\begin{array}{l}\text { Russian: } \\
50 \text { in cities } \\
65 \text { suburban } \\
80 \text { intercity } \\
\text { Foreign: } \\
60 \text { in cities } \\
80 \text { suburban } \\
105 \text { intercity }\end{array}$ \\
\hline NIIAT (2008) & $\begin{array}{l}14-16 \text { owned by } \\
\text { individuals } \\
25-30 \text { owned by } \\
\text { companies }\end{array}$ & & $30-40$ & $40-50$ \\
\hline $\begin{array}{l}\text { Avtostat } \\
(2010)\end{array}$ & $\begin{array}{l}15.3 \text { Russian } \\
18 \text { foreign-made }\end{array}$ & 55 & 63 & 65 \\
\hline $\begin{array}{l}\text { ICCT } \\
(2015)\end{array}$ & 10 & 10 & $13-38$ & 56 \\
\hline
\end{tabular}

Table S5. Average number kilometers traveled by type of vehicles.

\begin{tabular}{|c|c|c|c|}
\hline Vehicle type & Subsector & Emission standard & $\begin{array}{c}\text { Annual } \\
\text { kilometers } \\
\text { traveled }\end{array}$ \\
\hline & Diesel 1,4 - 2,0 1 & Conventional & 10000 \\
\hline & Diesel 1,4 - 2,01 & PC Euro 1 - 91/441/EEC & 10000 \\
\hline Passenger Cars & Diesel 1,4 - 2,0 1 & PC Euro 2 - 94/12/EEC & 15000 \\
\hline & Diesel 1,4 - 2,0 1 & PC Euro 3 - 98/69/EC Stage2000 & 15000 \\
\hline & Diesel 1,4 - 2,0 1 & PC Euro 4 - 98/69/EC Stage2005 & 18000 \\
\hline
\end{tabular}




\begin{tabular}{|c|c|c|c|}
\hline & Diesel 1,4 - 2,0 1 & PC Euro 5 - EC 715/2007 & 20000 \\
\hline \multirow{6}{*}{$\begin{array}{c}\text { Light } \\
\text { Commercial } \\
\text { Vehicles }\end{array}$} & Diesel $<3,5 \mathrm{t}$ & Conventional & 37000 \\
\hline & Diesel $<3,5 \mathrm{t}$ & LD Euro 1 - 93/59/EEC & 37000 \\
\hline & Diesel $<3,5 \mathrm{t}$ & LD Euro 2 - 96/69/EEC & 55000 \\
\hline & Diesel $<3,5 \mathrm{t}$ & LD Euro 3 - 98/69/EC Stage2000 & 55000 \\
\hline & Diesel $<3,5 \mathrm{t}$ & LD Euro 4 - 98/69/EC Stage2005 & 66000 \\
\hline & Diesel $<3,5 \mathrm{t}$ & LD Euro 5 - 2008 Standards & 73000 \\
\hline \multirow{24}{*}{$\begin{array}{c}\text { Heavy Duty } \\
\text { Trucks }\end{array}$} & Rigid $<=7,5 \mathrm{t}$ & Conventional & 42000 \\
\hline & Rigid $<=7,5 \mathrm{t}$ & HD Euro I - 91/542/EEC Stage I & 42000 \\
\hline & Rigid $<=7,5 \mathrm{t}$ & HD Euro II - 91/542/EEC Stage II & 63000 \\
\hline & Rigid $<=7,5 \mathrm{t}$ & HD Euro III - 2000 Standards & 63000 \\
\hline & Rigid $<=7,5 \mathrm{t}$ & HD Euro IV - 2005 Standards & 75000 \\
\hline & Rigid $<=7,5 \mathrm{t}$ & HD Euro V - 2008 Standards & 84000 \\
\hline & Rigid 7,5 - $12 \mathrm{t}$ & Conventional & 42000 \\
\hline & Rigid 7,5 - $12 \mathrm{t}$ & HD Euro I - 91/542/EEC Stage I & 42000 \\
\hline & Rigid 7,5 - $12 \mathrm{t}$ & HD Euro II - 91/542/EEC Stage II & 63000 \\
\hline & Rigid 7,5 - $12 \mathrm{t}$ & HD Euro III - 2000 Standards & 63000 \\
\hline & Rigid 7,5 - $12 \mathrm{t}$ & HD Euro IV - 2005 Standards & 75000 \\
\hline & Rigid 7,5 - $12 \mathrm{t}$ & HD Euro V - 2008 Standards & 84000 \\
\hline & Rigid $12-14$ t & Conventional & 42000 \\
\hline & Rigid $12-14 \mathrm{t}$ & HD Euro I - 91/542/EEC Stage I & 42000 \\
\hline & Rigid $12-14$ t & HD Euro II - 91/542/EEC Stage II & 63000 \\
\hline & Rigid $12-14$ t & HD Euro III - 2000 Standards & 63000 \\
\hline & Rigid $12-14$ t & HD Euro IV - 2005 Standards & 75000 \\
\hline & Rigid $12-14$ t & HD Euro V - 2008 Standards & 84000 \\
\hline & Rigid $14-20 t$ & Conventional & 42000 \\
\hline & Rigid $14-20 t$ & HD Euro I - 91/542/EEC Stage I & 42000 \\
\hline & Rigid $14-20 t$ & HD Euro II - 91/542/EEC Stage II & 63000 \\
\hline & Rigid $14-20 t$ & HD Euro III - 2000 Standards & 63000 \\
\hline & Rigid $14-20 t$ & HD Euro IV - 2005 Standards & 75000 \\
\hline & $\underline{\text { Rigid } 14-20 t}$ & HD Euro V - 2008 Standards & 84000 \\
\hline \multirow{11}{*}{ Buses } & Urban Buses Midi <=15 t & Conventional & 43000 \\
\hline & Urban Buses Midi $<=15 \mathrm{t}$ & HD Euro I - 91/542/EEC Stage I & 43000 \\
\hline & Urban Buses Midi <=15 t & HD Euro II - 91/542/EEC Stage II & 65000 \\
\hline & Urban Buses Midi <=15 t & HD Euro III - 2000 Standards & 65000 \\
\hline & Urban Buses Midi $<=15 \mathrm{t}$ & HD Euro IV - 2005 Standards & 78000 \\
\hline & Urban Buses Midi $<=15 \mathrm{t}$ & HD Euro V - 2008 Standards & 87000 \\
\hline & Urban Buses Standard $15-18 \mathrm{t}$ & Conventional & 43000 \\
\hline & Urban Buses Standard $15-18 \mathrm{t}$ & HD Euro I - 91/542/EEC Stage I & 43000 \\
\hline & Urban Buses Standard $15-18 \mathrm{t}$ & HD Euro II - 91/542/EEC Stage II & 65000 \\
\hline & Urban Buses Standard $15-18 \mathrm{t}$ & HD Euro III - 2000 Standards & 65000 \\
\hline & Urban Buses Standard $15-18 \mathrm{t}$ & HD Euro IV - 2005 Standards & 78000 \\
\hline
\end{tabular}


Urban Buses Standard 15 - 18 t HD Euro V - 2008 Standards $\quad 87000$

Urban Buses Articulated $>18 \mathrm{t}$ Conventional 43000

Urban Buses Articulated >18 t HD Euro I - 91/542/EEC Stage I 43000

Urban Buses Articulated >18 t HD Euro II - 91/542/EEC Stage II 65000

Urban Buses Articulated >18 t HD Euro III - 2000 Standards $\quad 65000$

Urban Buses Articulated >18 t HD Euro IV - 2005 Standards $\quad 78000$

Urban Buses Articulated >18 t HD Euro V - 2008 Standards $\quad 87000$ 


\section{Results of emission calculations}

The COPERT 4 model with NIIAT emission factors. The assumptions on average annual kilometers traveled remain the same.

Table S6. OC emissions from the adjusted fleet with superemitters ( $t$ ).

\begin{tabular}{lccccc}
\hline & Cars & LCVs & Trucks & Buses & Total \\
\hline Euro 0 & 86 & 577 & 3253 & 184 & 4100 \\
Euro 1 & 21 & 125 & 153 & 39 & 338 \\
Euro 2 & 25 & 107 & 478 & 84 & 694 \\
Euro 3 & 17 & 78 & 466 & 98 & 659 \\
Euro 4 & 28 & 121 & 85 & 10 & 244 \\
Euro 5 & 13 & 5 & 28 & 1 & 46 \\
Total & 190 & 1012 & 4463 & 415 & 6080 \\
\hline
\end{tabular}

Table S7. BC emissions, assuming that all trucks and buses use diesel fuel (t).

\begin{tabular}{|c|c|c|c|c|c|}
\hline & Cars & LCVs & Trucks & Buses & Total \\
\hline Euro 0 & & & 30742 & 2687 & 33429 \\
\hline Euro 1 & & & 1005 & 238 & 1243 \\
\hline Euro 2 & & & 2346 & 584 & 2930 \\
\hline Euro 3 & & & 2200 & 638 & 2837 \\
\hline Euro 4 & & & 384 & 63 & 447 \\
\hline Euro 5 & & & 110 & 3 & 113 \\
\hline Total & & & 36788 & 4212 & 41000 \\
\hline
\end{tabular}

Table S8. BC emissions from all registered diesel vehicles ( $t$ ).

\begin{tabular}{lccccr}
\hline & Cars & LCVs & Trucks & Buses & Total \\
\hline Euro 0 & 330 & 1750 & 14248 & 663 & 16990 \\
Euro 1 & 92 & 545 & 1004 & 203 & 1844 \\
Euro 2 & 162 & 683 & 2167 & 325 & 3337 \\
Euro 3 & 144 & 654 & 2138 & 420 & 3356 \\
Euro 4 & 242 & 988 & 383 & 45 & 1658 \\
Euro 5 & 6 & 2 & 110 & 3 & 122 \\
Total & 976 & 4622 & 20051 & 1658 & 27307 \\
\hline
\end{tabular}


Table S9. BC emissions from the adjusted diesel fleet without accounting for superemitters (t).

\begin{tabular}{|c|c|c|c|c|c|}
\hline & Cars & LCVs & Trucks & Buses & Total \\
\hline Euro 0 & 181 & 1225 & 5699 & 331 & 7437 \\
\hline Euro 1 & 69 & 409 & 502 & 122 & 1102 \\
\hline Euro 2 & 130 & 546 & 1300 & 227 & 2203 \\
\hline Euro 3 & 123 & 556 & 1604 & 336 & 2617 \\
\hline Euro 4 & 45 & 939 & 345 & 40 & 1369 \\
\hline Euro 5 & 6 & 2 & 110 & 3 & 122 \\
\hline Total & 554 & 3677 & 9560 & 1060 & 14851 \\
\hline
\end{tabular}

\section{References}

Avtostat: Park of PC, LCV, HCV, BUS as of 01. 01.2015 (Russia, Russian regions). Dataset, Avtostat, Togliatti, Russia, 2015.

Emisia: COPERT (COmputer Programme to Calculate Emissions from Road Transport).

Prepared for the European Environment Agency, Available at http://www.emisia.com/copert/ (last access:15 July), 2015.

ICCT: Global Transportation Roadmap Model International Council on Clean Transportation, Available at http://www.theicct.org/global-transportation-roadmap-model (accessed September 15, 2015), 2015.

NIIAT: The assessment methodology for estimating the residual value of vehicles with regard to technical condition, Scientific Research Institute of Automobiles and Transportation, Moscow, 1998.

NIIAT: Calculation instruction (methodology) for emissions inventory from vehicles on the territory of the largest cities (in Russian), Scientific Research Institute of Automobiles and Transportation, Moscow, 2012. 